Research Article

\title{
Simulation of Spatially Correlated Multipoint Ground Motions in a Saturated Alluvial Valley
}

\author{
Ying He $\mathbb{C}^{1,2}$ Xueling Chen, ${ }^{1}$ Zhongxian Liu, ${ }^{1,2}$ Dejian Yang, ${ }^{1}$ and Hai Zhang ${ }^{1}$ \\ ${ }^{1}$ Tianjin Key Laboratory of Civil Structure Protection and Reinforcement, Tianjin Chengjian University, Tianjin 300384, China \\ ${ }^{2}$ Earthquake Engineering Research Institute of Tianjin City, Tianjin 300074, China \\ Correspondence should be addressed to Ying He; he114@126.com
}

Received 16 June 2021; Accepted 3 September 2021; Published 22 September 2021

Academic Editor: Vadim Silberschmidt

Copyright $(02021$ Ying He et al. This is an open access article distributed under the Creative Commons Attribution License, which permits unrestricted use, distribution, and reproduction in any medium, provided the original work is properly cited.

Based on Biot's theory, the boundary element method, and spectral representation method, an effective simulation method for multiple-station spatially correlated ground motions on both bedrock and surface is developed, incorporating the spectral density function, coherence function, and site transfer function that consider both the wave scattering effect and the medium saturation. The accuracy and feasibility of the present method are validated by a typical numerical example. Our results indicate that the local site conditions and the saturation property of the medium significantly affect the multipoint spatially correlated earthquake ground motions, especially in the long-period range. It is necessary to use spatially varying ground motions with the rational consideration of local site effects and medium saturation as input during the seismic analysis of large-span structures.

\section{Introduction}

Many cities are located on alluvial valleys, and numerous seismic observations and damage surveys have confirmed the significant influence of alluvial valleys on ground motion [1-5]. A typical example is a great deal of large-span bridges and high dams built in saturated alluvial valleys. However, the dynamic response of large-span structures is greatly affected by the seismic spatial variation effect, which may lead to errors in calculation. Accurate and reliable ground motion parameters are needed in the seismic design of important engineering structures. Therefore, the influence of multiple input conditions should be considered when studying the seismic response of large-span structures [6]. In a word, the multistation ground motion in a saturated alluvial valley studied in this paper has a profound influence on the theoretical research value and engineering application of future seismic research and development directions.

Theoretically, the choice of power spectral function and coherence function model is the key to simulate spatially varying seismic motions. Furthermore, many scholars have proposed several coherency function models to simulate spatially varying seismic ground motions, such as those reported by Loh, Feng et al., Hao et al., Harichandran et al., and $\mathrm{Qu}$ et al. [7-11]. They obtained the ground motion data of the dense array by statistical regression using stochastic signal processing techniques. Some scholars [12-14] established the model of coherence function from theoretical analysis and then fitted the parameters in the model based on ground motion records. Kiureghian attributed the spatial variation of ground motion to the Wave Passage Effect, Incoherence Effect, Attenuation Effect, and Site Response Effect. Studying the influence of a single factor makes the analysis of ground motion more in line with the actual engineering site.

The key point of constructing the power spectrum function is the calculation of the transfer function. The current research [15-17] calculated the transfer function mostly based on the one-dimensional wave propagation theory [18] of layered half-space medium, without considering the scattering effect of seismic waves at local sites and the saturation state of the medium. The scattering effect of local topography on seismic waves cannot be ignored, and the medium in the valley site is often in a saturation state, which is quite different from the usual single-phase elastic model. Many scholars have studied seismic wave scattering 
[19-26]. It is worth pointing out that the above studies all focus on the seismic response of the alluvial valleys with elastic media. However, it should be noted that saturated soil is a kind of natural soil that is common in the coastal and valley areas, and the foundation soil of many cities is saturated, such as Tianjin, China. Based on Biot's theory [27] of wave propagation in saturated soil, the propagation characteristics of seismic waves in saturated poroelastic medium are quite different from those in the elastic medium model. It is more practical to use the saturated model to study it. Due to the complexity of the problem, there are relatively few studies based on saturated porous media models. Lin et al. $[28,29]$ discussed the dynamic response of plane wave reflection in saturated porous half space. Li et al. [30-33] gave the analytical solutions of $\mathrm{P}$ and SV wave scattering in semicircular saturated depressions and saturated alluvial valleys. The scattering law of Pwave around a canyon was studied by $\mathrm{Ba}$ et al. [34] using the wave function expansion method. Liu [35] used the indirect boundary element method (IBEM) to study the scattering of $\mathrm{P}$ waves in saturated alluvial valleys. The above papers also illustrate the necessity of considering the wave scattering effect in the multistation seismic motion simulation. He et al. [36, 37] considered the seismic wave scattering of complex terrain in elastic medium but did not consider the saturated medium. Based on the above literature, it can be seen that spatially related ground motion simulation considering saturated media is rare and still has room for development.

So far, many scholars [38-45] have studied spatially correlated seismic motions with local field effects in mind. At present, there are mainly two methods of spatially related multipoint ground motion simulation that have a great influence. The first method is based on the assumption of stationary random process and reflects the correlation of all points in space through the ground motion power spectrum matrix, and the representative one is the HOP [9] method. The second method is to use the random field interpolation method to obtain the time history of ground motion at other points. The representative method is Vanmarcke et al.'s [46] method.

In this study, an effective method is proposed to simulate spatially correlated ground motions at multiple stations by considering the scattering effects of seismic waves at local sites, such as saturated alluvial valley sites. Among all kinds of numerical methods, the boundary element method [30] (BEM) chosen in this paper is more suitable for calculating the broadband frequency of two-dimensional transfer function in half space. It has the unique advantage of reducing the dimension of the problem and automatically satisfying the infinite boundary conditions, which is particularly obvious in this paper. Therefore, considering the spatial coherence effect, wave scattering effect, and saturated medium comprehensively, the BEM combined with the prototype spectral representation method [9] (PSRM) was adopted to obtain multistation ground motion in the saturated alluvial valley site. The method presented in this paper provides a new idea for the scattering of elastic waves in saturated media, which has important theoretical significance and engineering application, and provides a certain theoretical basis for the seismic design of engineering projects and the scientific basis of urban planning.

\section{Method}

2.1. Calculation Model and Method. The model used in this paper is shown in Figure 1. In the saturated half space, there is an alluvial valley with the shape of a semicircle. jand $k$ are any two points on the overburden, and $j^{\prime}$ and $k^{\prime}$ are the corresponding points on the hypothetical bedrock surface. The excitation is assumed to be incident at an angle $\alpha$ from the bedrock half space.

\subsection{Multipoint Ground Motion Calculation Method.} Because the distance from the seismic source to the surface is much larger than the size of the alluvial valley, it can be assumed that the ground motion at the bedrock is a zeromean stationary random process with the same intensity and frequency components. In this paper, the Clough-Penzien model [47] is used to simulate the power spectral density function.

The spatially related auto and cross power spectrum can be expressed as

$$
\begin{aligned}
& S_{j j}(\omega)=\left|H_{j}(i \omega)\right|^{2} S_{g}(\omega), \\
& S_{j k}(i \omega)=H_{j}(i \omega) H_{k}^{*}(i \omega) S_{g}(\omega)\left|\gamma_{j^{\prime} k^{\prime}}\left(d_{j^{\prime} k^{\prime}}, i \omega\right)\right| e^{-i \omega d_{j^{\prime} k^{\prime}} \cos \alpha / v_{a p p}} \quad j, k=1,2, \ldots, n,
\end{aligned}
$$

where $H_{j}(i \omega)$ and $H_{k}(i \omega)$ are the transfer functions at locations jand $k$, which reflect the influence of local soil on wave propagation. The symbol *represents the complex conjugate. $\left|\gamma_{j^{\prime} k^{\prime}}(i \omega)\right|$ denotes the lagged coherency, which reveals the influence of valley topography on the spatial correlation of ground motion. The Sobczyk model (Sobczky, 1991) is used in this study.
Therefore, the power spectrum density functions of spatial ground motion at the bedrock can be expressed as a positive definite Hermite matrix $\mathbf{S}(i \omega)$, which can be simplified by Cholesky decomposition.

$$
\mathbf{S}(i \omega)=\mathbf{L}(i \omega)\left[\mathbf{L}^{*}(i \omega)\right]^{T}
$$




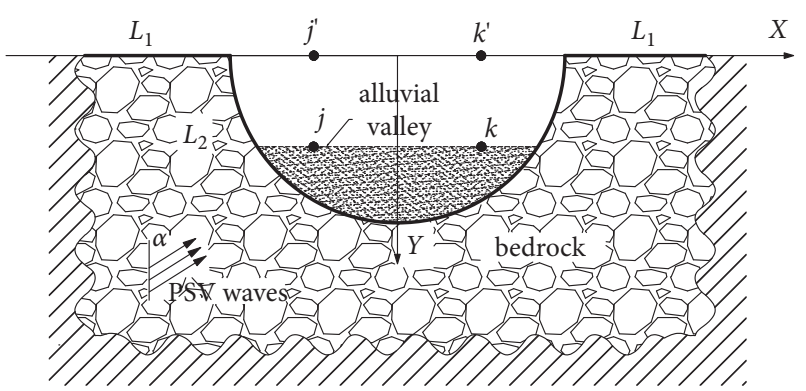

FIgURe 1: Model diagram.

According to PSRM, the stationary time histories can be directly expressed as

$$
q_{j}(t)=\sum_{k=1}^{j} \sum_{i=1}^{N} A_{j k}\left(\omega_{i}\right) \cos \left[\omega_{i} t+\theta_{j k}\left(\omega_{i}\right)+\varphi_{k i}\left(\omega_{i}\right)\right]
$$

where

$$
\begin{aligned}
& A_{j k}(\omega)=\sqrt{4 \Delta \omega}\left|L_{j k}(\omega)\right|, \\
& \theta_{j k}(\omega)=\tan ^{-1} \frac{\operatorname{Im}\left[L_{j k}(\omega)\right]}{\operatorname{Re}\left[L_{j k}(\omega)\right]},
\end{aligned}
$$

are the amplitudes and phase angles of the simulated time histories.

To obtain a nonstationary time histories that is consistent with the actual ground motion, an envelope function $f(t)$ is applied to $q_{j}(t)$.

$$
x_{j}(t)=f(t) \cdot q_{j}(t) .
$$

\subsection{Transfer Function Calculated by Biot's Saturated Poroelastic Medium Theory and Boundary Element Method}

2.3.1. Biot's Theory. According to Biot's saturated poroelastic medium theory [48], the constitutive equation of saturated medium is as follows:

$$
\begin{aligned}
\sigma_{i j} & =\lambda e \delta_{i j}+2 \mu \varepsilon_{i j}-\delta_{i j} \alpha P(i, j=x, y), \\
P & =-\alpha M u_{i, i}-M w_{i, i},
\end{aligned}
$$

where $\sigma_{i j}$ and Prepresent the total stress component and pore pressure of soil, respectively; $e$ and $\varepsilon_{i j}$, respectively, represent the volumetric strain and strain component of soil skeleton; $\lambda$ and $\mu$ are the Lame constant of soil skeleton; $\delta_{i j}$ is the Kronecker function, with a value of 0 or 1 ; and $\alpha$ and $M$ are parameters used to indicate the compressibility of soil particles and pore fluids $(0 \leq \alpha \leq 1,0 \leq M \leq \infty)$.

The solid frame displacement and fluid displacement relative to solid frame are denoted by $u_{i}$ and $w_{i}$, and the motion equations in saturated poroelastic medium can be expressed as follows [49]:

$$
\begin{aligned}
\mu u_{i, j j}+\left(\lambda+\alpha^{2} M+\mu\right) u_{j, j i}+\alpha M w_{j, j i} & =\rho \ddot{u}_{i}+\rho_{f} \ddot{u}_{i}, \\
\alpha M u_{j, j i}+M w_{j, j i} & =\rho_{f} \ddot{u}_{i}+m \ddot{w}_{i}+b \dot{w}_{i},
\end{aligned}
$$

where $\rho$ is the total density of soil, $\rho_{f}$ is the mass density of fluid, $m$ is a parameter corresponding to the physical meaning of mass, and $b$ is the coefficient used to reflect the degree of viscosity coupling of soil. If internal friction is not considered, then $b=0$.

2.3.2. Free Field. The free field displacement is calculated by using the wave reflection principle; when the SV wave is incident at an angle $\theta_{\beta}$, three kinds of reflected waves ( $\mathrm{P}_{\mathrm{I}}$ wave, $\mathrm{P}_{\mathrm{II}}$ wave, and $\mathrm{SV}$ wave) are generated on the reflective surface of the ground in the saturated half space. Then, the expression of the potential functions of the above three waves in the Cartesian coordinate system is as follows:

$$
\begin{aligned}
& \phi_{1}^{r}=a_{1} e^{-i k_{\alpha 1}\left(x \sin \theta_{\alpha 1}+y \cos \theta_{\alpha 1}\right)}, \\
& \phi_{2}^{r}=a_{2} e^{-i k_{\alpha 2}\left(x \sin \theta_{\alpha 2}+y \cos \theta_{\alpha 2}\right)} . \\
& \psi^{r}=b_{1} e^{-i k_{\beta}\left(x \sin \theta_{\beta}+y \cos \theta_{\beta}\right)},
\end{aligned}
$$

where $a_{1}, a_{2}$, and $b_{1}$ represent the reflection coefficients and $k_{\alpha 1}, k_{\alpha 2}$, and $k_{\beta}$ represent the wave number. According to the relationship between the potential function and the displacement, solid frame displacement $u_{i}^{(f)}$, fluid relative displacement $w_{i}^{(f)}$, and pore pressure $p^{(\pi)}$ in the free field can be, respectively, expressed as follows:

$$
\begin{aligned}
& u_{x}^{(f)}=-i k_{\alpha 1}\left(\phi_{1}^{(r)}+\phi_{2}^{(r)}\right) \sin \theta_{\alpha 1}+i k_{\beta}\left(\psi^{(i)}-\psi^{(r)}\right) \cos \theta_{\beta}, \\
& u_{y}^{(f)}=i k_{\beta}\left(\psi^{(i)}+\psi^{(r)}\right) \sin \theta_{\beta}-i k_{\alpha 1} \phi_{1}^{(r)} \cos \theta_{\alpha 1}-i k_{\alpha 2} \phi_{2}^{(r)} \cos \theta_{\alpha 2}, \\
& w_{x}^{(f)}=-i k_{\alpha 1}\left[\chi_{1} \phi_{1}^{(r)}+\chi_{2} \phi_{2}^{(r)}\right] \sin \theta_{\alpha 1}+i k_{\beta} \chi_{3}\left(\psi^{(i)}-\psi^{(r)}\right) \cos \theta_{\beta}, \\
& w_{y}^{(f)}=i k_{\beta}\left[\chi_{3}\left(\psi^{(i)}+\psi^{(r)}\right)\right] \sin \theta_{\beta}-i k_{\alpha 1} \chi_{1} \phi_{1}^{(r)} \cos \theta_{\alpha 1}-i k_{\alpha 2} \chi_{2} \phi_{2}^{(r)} \cos \theta_{\alpha 2}, \\
& p^{(f)}=\left(\alpha+\chi_{1}\right) M k_{\alpha 1}^{2} \phi_{1}+\left(\alpha+\chi_{2}\right) M k_{\alpha 2}^{2} \phi_{2} .
\end{aligned}
$$


2.3.3. Scattering Field. Scattered waves will be generated in half space and inside the sedimentary due to the presence of alluvial valleys. According to the single-layer potential theory, the scattering wave can be formed by the superposition of the expansion wave sources and shear wave sources on the virtual wave source surface inside and outside the deposition.

$$
\begin{aligned}
& u_{i}^{s}(x)=b_{n^{\prime}} G_{i, 1}^{(s)}\left(x_{n}, x_{n^{\prime}}\right)+c_{n^{\prime}} G_{i, 2}^{(s)}\left(x_{n}, x_{n^{\prime}}\right)+d_{n^{\prime}} G_{i, 3}^{(s)}\left(x_{n}, x_{n^{\prime}}\right), \\
& w_{i}^{s}(x)=b_{n^{\prime}} G_{w i, 1}^{(s)}\left(x_{n}, x_{n^{\prime}}\right)+c_{n^{\prime}} G_{w i, 2}^{(s)}\left(x_{n}, x_{n^{\prime}}\right)+d_{n^{\prime}} G_{w i, 3}^{(s)}\left(x_{n}, x_{n^{\prime}}\right), \\
& \sigma_{i j}^{s}(x)=b_{n^{\prime}} T_{i j, 1}^{(s)}\left(x_{n}, x_{n^{\prime}}\right)+c_{n^{\prime}} T_{i j, 2}^{(s)}\left(x_{n}, x_{n^{\prime}}\right)+d_{n^{\prime}} T_{i j, 3}^{(s)}\left(x_{n}, x_{n^{\prime}}\right), \\
& p^{s}\left(x_{n}\right)=b_{n^{\prime}} T_{p, 1}^{(s)}\left(x_{n}, x_{n^{\prime}}\right)+c_{n^{\prime}} T_{p, 2}^{(s)}\left(x_{n}, x_{n^{\prime}}\right)+d_{n^{\prime}} T_{p, 3}^{(s)}\left(x_{n}, x_{n^{\prime}}\right),
\end{aligned}
$$

where $n /$ is the point on the virtual source plane; $b_{n}, c_{n}$, and $d_{n}$ are the source densities of $\mathrm{P}_{\mathrm{I}}$ wave, $\mathrm{P}_{\mathrm{II}}$ wave, and SV wave at the $n$ 'discrete point on the virtual source plane, respectively; $G_{i, l}^{(s)}\left(x_{n}, x_{n^{\prime}}\right), \quad G_{i, l}^{(s)}\left(x_{n}, x_{n^{\prime}}\right), \quad T_{i, l}^{(s)}\left(x_{n}, x_{n^{\prime}}\right), \quad$ and $T_{l}^{(s)}\left(x_{n}, x_{n^{\prime}}\right)$, respectively, represent Green's functions of solid frame displacement, fluid relative displacement, total stress, and pore pressure in saturated half space (subscript $l=1,2,3$ corresponding to $\mathrm{P}_{\mathrm{I}}$ wave, $\mathrm{P}_{\mathrm{II}}$ wave, and $\mathrm{SV}$ wave sources, respectively).

According to IBEM and elastic wave theory, the total displacement of any point in the half space can be obtained as follows:

$$
u^{(t)}=u^{(s)}+u^{(f)}
$$

where $u^{(s)}$ is the scattered wave field constructed by the virtual wave source and $u^{(f)}$ represents the free field displacement at any point.

Assuming that there is a vertical transfer relationship exists between the ground motion of the alluvial valley points and the imaginary bedrock surface points, then the transfer function can be expressed as

$$
H_{j}(\omega)=\frac{u_{j}(\omega)}{u_{j^{\prime}}(\omega)}
$$

2.3.4. Boundary Condition. Since the dynamic Green's functions in saturated half space automatically satisfy the free surface boundary condition, only the continuity conditions of the interface between saturated deposition and half space are considered. In the case of boundary permeability, the boundary conditions are as follows:

$$
\left\{\begin{array}{l}
u_{x}^{s}=u_{x}^{v}, u_{y}^{s}=u_{y}^{v}, w_{n}^{s}=w_{n}^{v} \\
\sigma_{n n}^{s}=\sigma_{n n}^{v}, \sigma_{n t}^{s}=\sigma_{n t}^{v} \\
p^{s}-p^{v}=0
\end{array}\right.
$$

where the superscripts sand vrepresent the half space and alluvial valley, respectively.

\section{Correctness Verification}

To verify the correctness of the method in this paper, a comparison between the surface displacement amplitude calculated in this paper and the results in the literature is given. In consideration of degradation, the calculated parameters are as follows: porosity is 0.001 , dimensionless frequency $\eta=\omega a / c_{\beta} \pi$, Poisson's ratio $\nu=1 / 3$, damping ratio $\zeta=0.001$, and incident angle $\theta_{\beta}=0^{\circ}$. The results presented in this paper are in good agreement with those of the corresponding elastic media in Figure 2(Sanchez-Sesma and Campillo [21]) and Figure 3(Dravinski and Mossessian [50]), thus verifying the correctness of the method presented in this paper.

\section{Numerical Examples}

The site model of a semicircular alluvial valley with permeable boundary is shown in Figure 4. Taking the SV wave incident at 30 degrees from the bedrock half space as an example, three points 1,2 , and 3 were selected along the horizontal ground. The spacing is shown in the figure. Points $1^{\prime}, 2^{\prime}$, and $3^{\prime}$ are, respectively, the corresponding points of the assumed horizontal field directly above the surface of the alluvial valley. Table 1shows the corresponding physical parameters of the soil layer, the fluid density is $1000 \mathrm{~kg} / \mathrm{m}^{3}$, and the selection of horizontal ground motion parameters as follows:

$$
\begin{aligned}
& \omega_{g}=10 \pi \frac{\mathrm{rad}}{\mathrm{s}}, \\
& \zeta_{g}=0.6, \\
& \omega_{f}=\frac{0.5 \pi \mathrm{rad}}{\mathrm{s}}, \\
& \zeta_{f}=0.6, \\
& S_{0}=\frac{0.0034 \mathrm{~m}^{2}}{\mathrm{~s}^{2}} .
\end{aligned}
$$



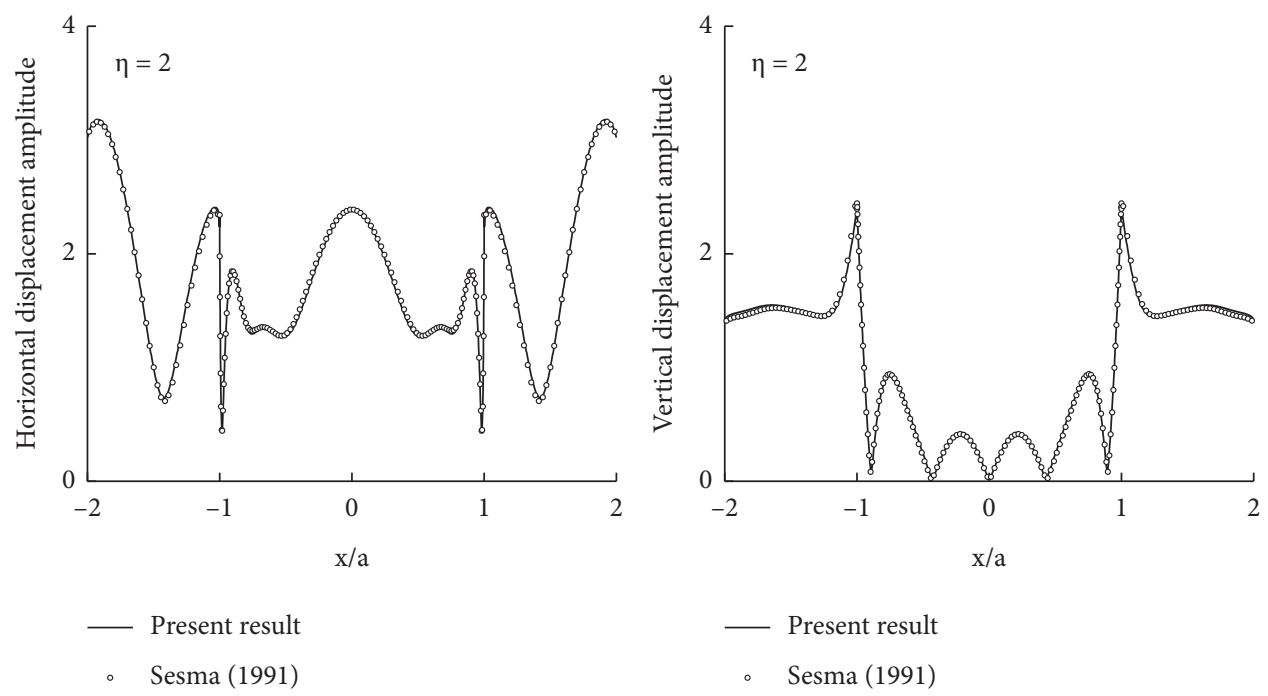

FIgURE 2: Comparison of the degenerated canyon results with the classical results.
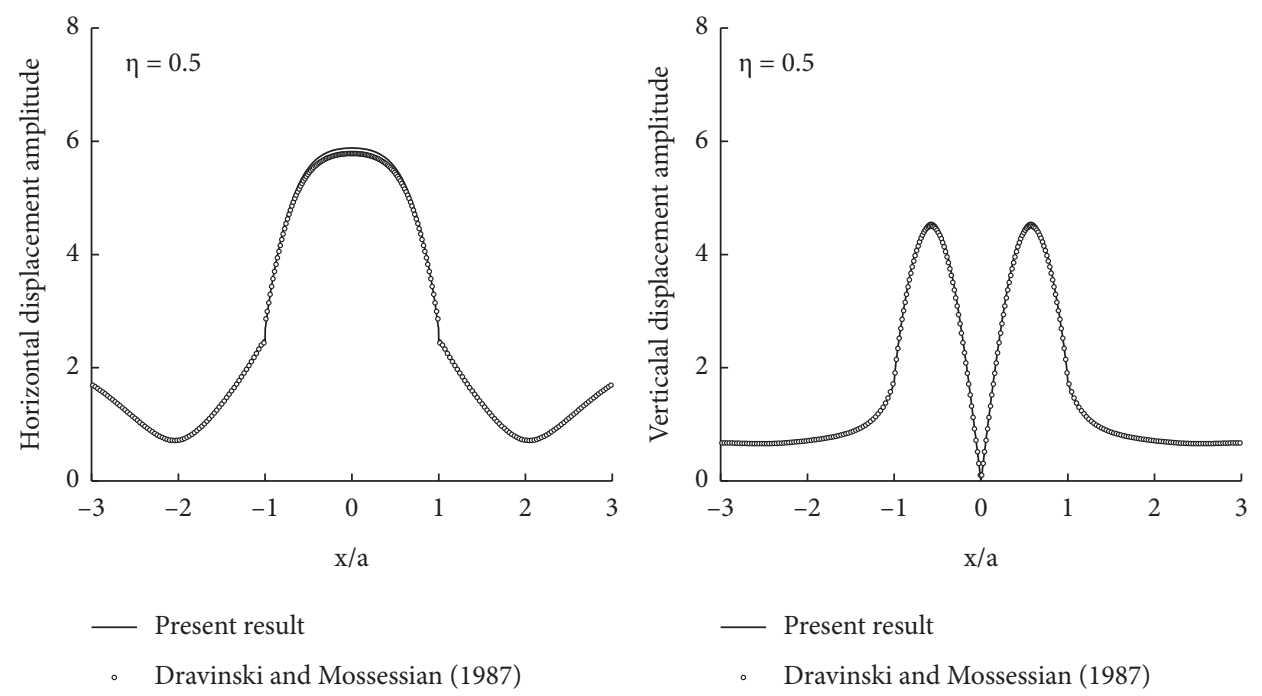

FIGURE 3: Comparison of the degenerated alluvial valley results with the classical results.

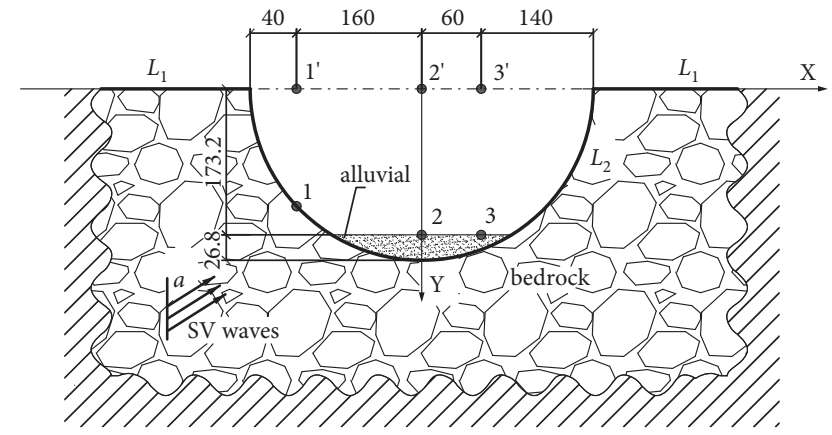

FIGURE 4: Model of a saturated semicircular alluvial valley.

To verify the simulation method, the auto/cross-PSDs (power spectrum densities) are compared with the corresponding theoretical values, which are obtained by equation (2). The auto/cross-PSD estimates of the simulated ground motions are obtained by averaging the estimates from 30 generated samples. Figure 5shows the comparisons of the auto/cross-PSDs of the generated acceleration time histories with the corresponding target ones of the alluvial valley. Not only the auto-PSDs of the simulated motions match well with those of the target spectrum but also the cross-PSDs of the simulated motions match well with those of the target spectrum. Therefore, the effectiveness of this method is illustrated.

Figure 6shows five sets of acceleration history samples of the saturated alluvial valley. For comparison, Figure 7 shows the corresponding equivalent elasticity. The response spectra obtained from samples of the two cases are given in Figure 8.

Table 2shows five sets of peak ground acceleration (PGA) samples and the peak response spectrum (PRS) of the two cases. PGA1 and PRS1 mean the PGA and PRS calculated by the method in this paper, respectively, while PGA2 and PRS2 
TABle 1: Parameters of soil layer.

\begin{tabular}{lcccccc}
\hline & Porosity & Critical porosity & Density $\left(\mathrm{kg} / \mathrm{m}^{3}\right)$ & Poisson's ratio & Shear wave velocity $(\mathrm{m} / \mathrm{s})$ & Damping ratio \\
\hline Alluvial & 0.3 & 0.36 & 2000 & 0.25 & 444.5 & 0.05 \\
Bedrock & 0.1 & 0.36 & 2000 & 0.25 & 708 & 0.02 \\
\hline
\end{tabular}
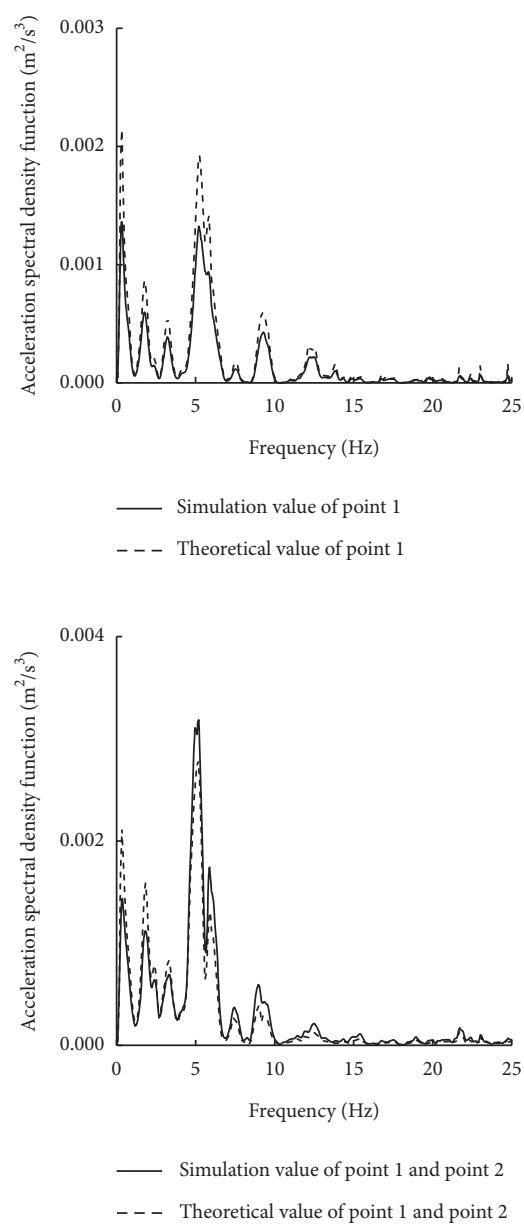

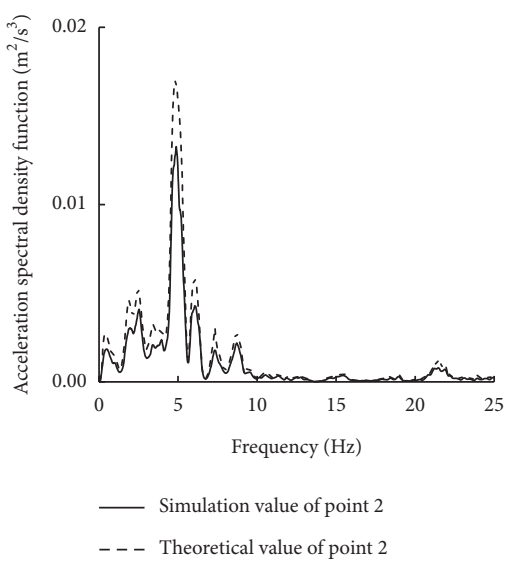

(a)
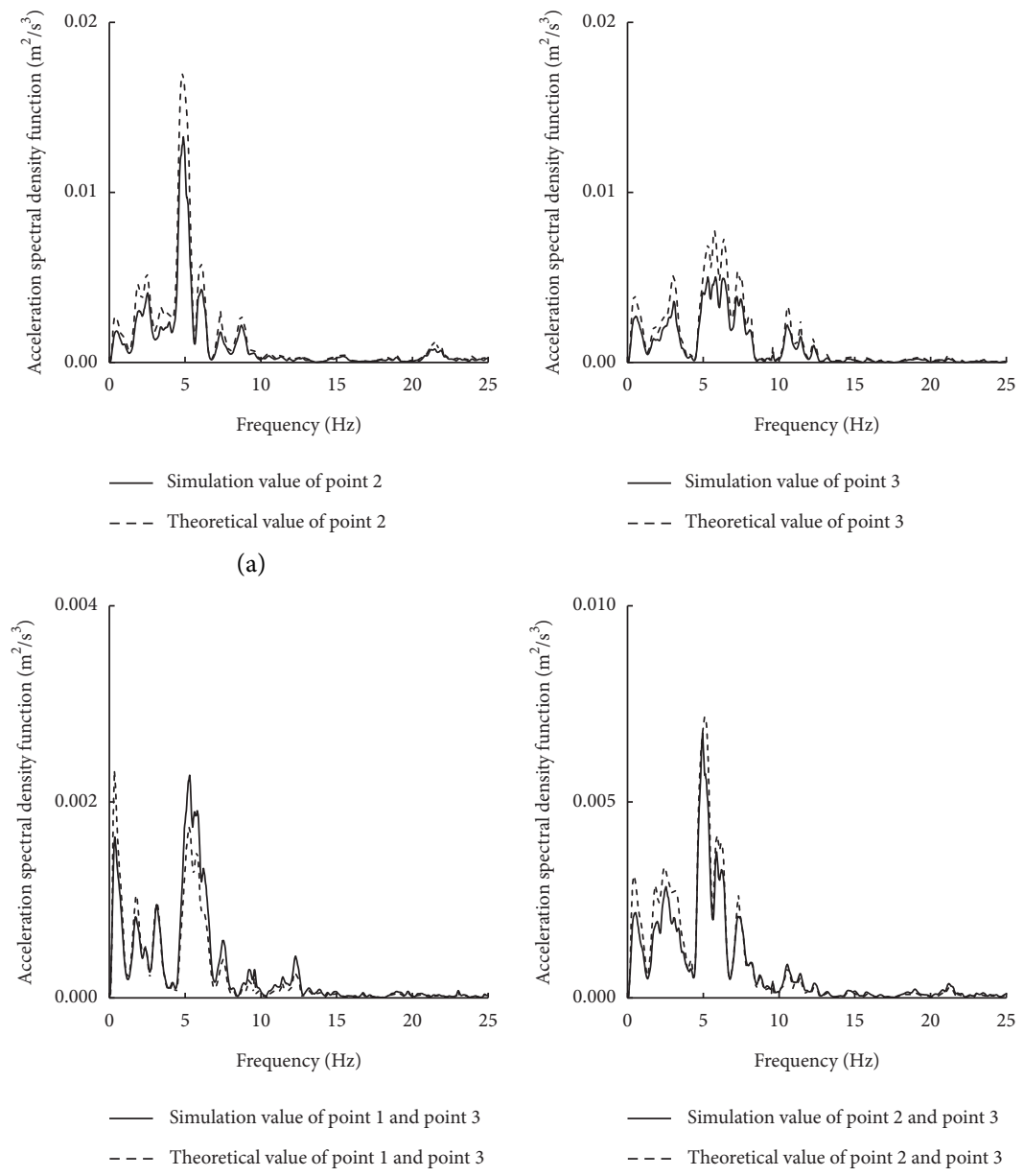

(b)

FIgURE 5: (a) Acceleration auto-PSD estimates from 30 sets of ground motions. (b) Acceleration cross-PSD estimates from 30 sets of ground motions.

mean the PGA and PRS calculated by the elastic method, respectively.

According to the numerical comparison in Table 2, it can be clearly seen that the heterogeneity of seismic spatial distribution in the alluvial valley is different from whether the saturation state of the alluvial valley is considered. From the perspective of spatial relationship, compared with 1 point, the acceleration amplitude of 2 point and 3 point is larger. Taking the mean value of PGA1 as an example, the acceleration amplitude of 2 point is 1.7 times more than that of 1 point, and the acceleration amplitude of 3 point is 1.46 times more than that of 1 point. This is mainly because point 1 is located outside the sedimentary overburden, namely, on the bedrock, which reflects the vibration characteristics of the input seismic wave more. The remaining two points are located in the alluvial valley, and the energy focusing and filtering and amplification of the seismic wave occur in the process of propagation. Therefore, the spatial effect of ground motion can not be ignored. For example, for a long and large structure across a saturated alluvial valley, the support points located in the inner part of the alluvial valley should be input more severe earthquake motion than those on the outer bedrock instead of the same motion. This is also the significance of this study, which provides a reasonable basis for the spatial correlation multipoint seismic input of local sites.

The saturation model and elastic model have great differences in peak acceleration, peak value of response spectrum, and predominant period of the response spectrum. According to the calculation results of the saturated model, the PGA at 1 point on the bedrock decreases by $44.7 \%$ compared with that of the elastic model and the PGA 

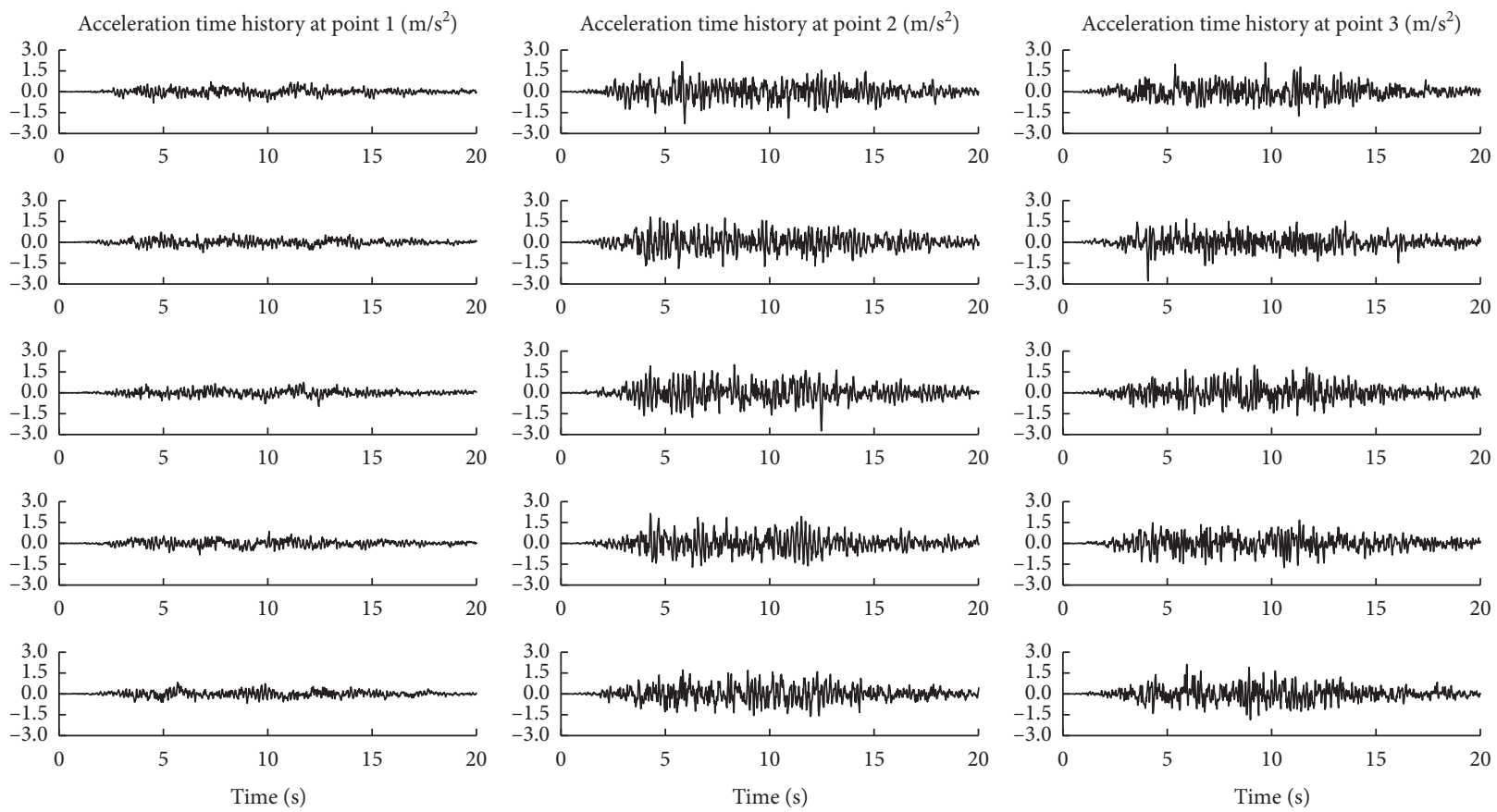

FIGURE 6: Samples of spatially correlated earthquake ground motion simulation (saturated alluvial valley).
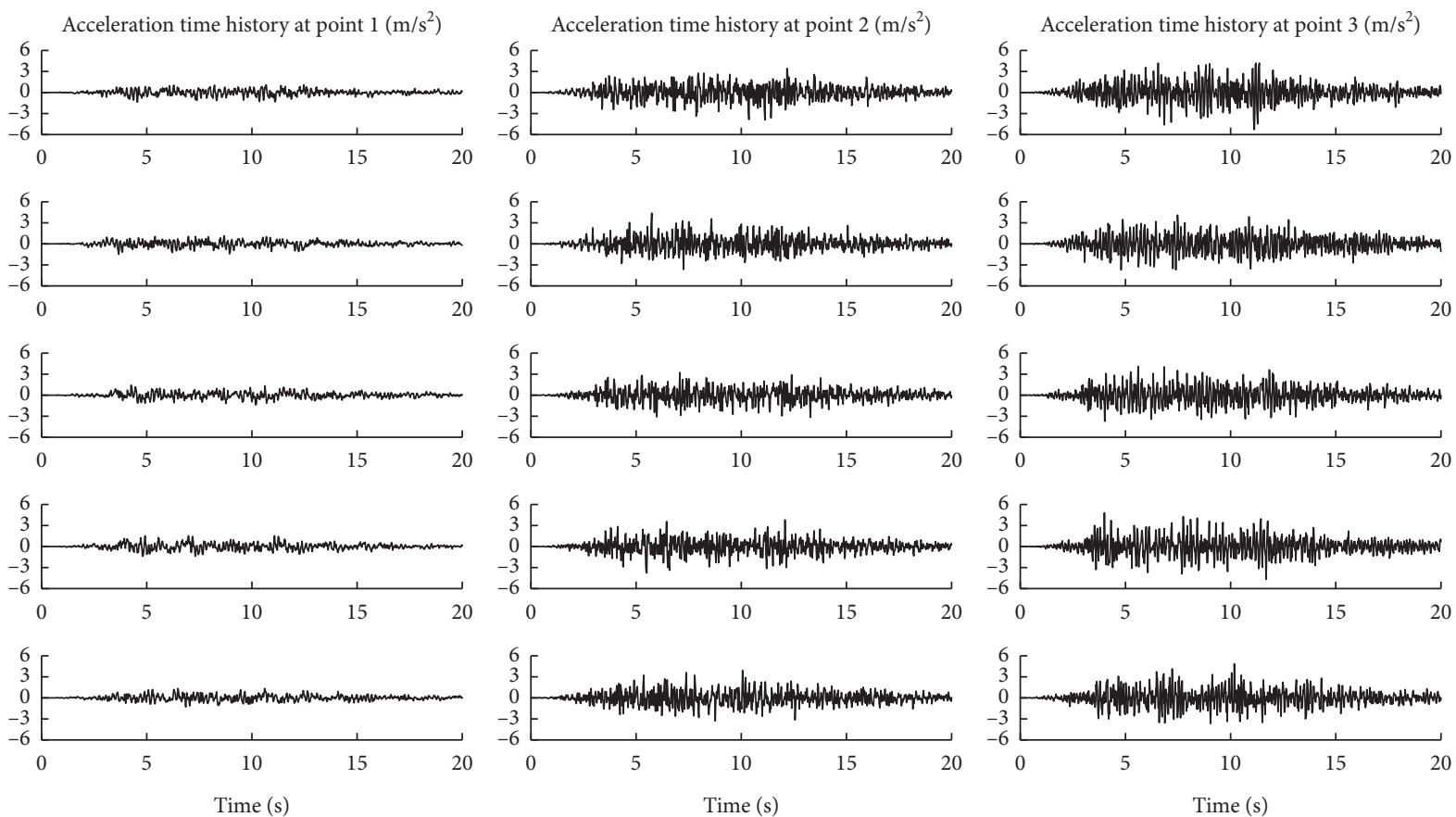

FIgURE 7: Samples of spatially correlated earthquake ground motion simulation (elastic alluvial valley).

at 2 points in the center of the sedimentary layer and 3 points near the edge decreases by $42.1 \%$ and $56.8 \%$, respectively. This is because the pore water dissipates the vibration energy greatly in the saturation medium, and the seismic response is reduced to some extent. This is also reflected in the peak of the reaction spectrum. It is worth noting that the predominant period corresponding to the peak value of the response spectrum is also different in the two cases: the 1point predominant period at bedrock has little change; but in the alluvial valley, the high frequency response in saturated condition is much less than that in elastic condition, and the predominant period extends from $0.09 \mathrm{~s}$ to $0.18 \mathrm{~s}$ at 2 point 

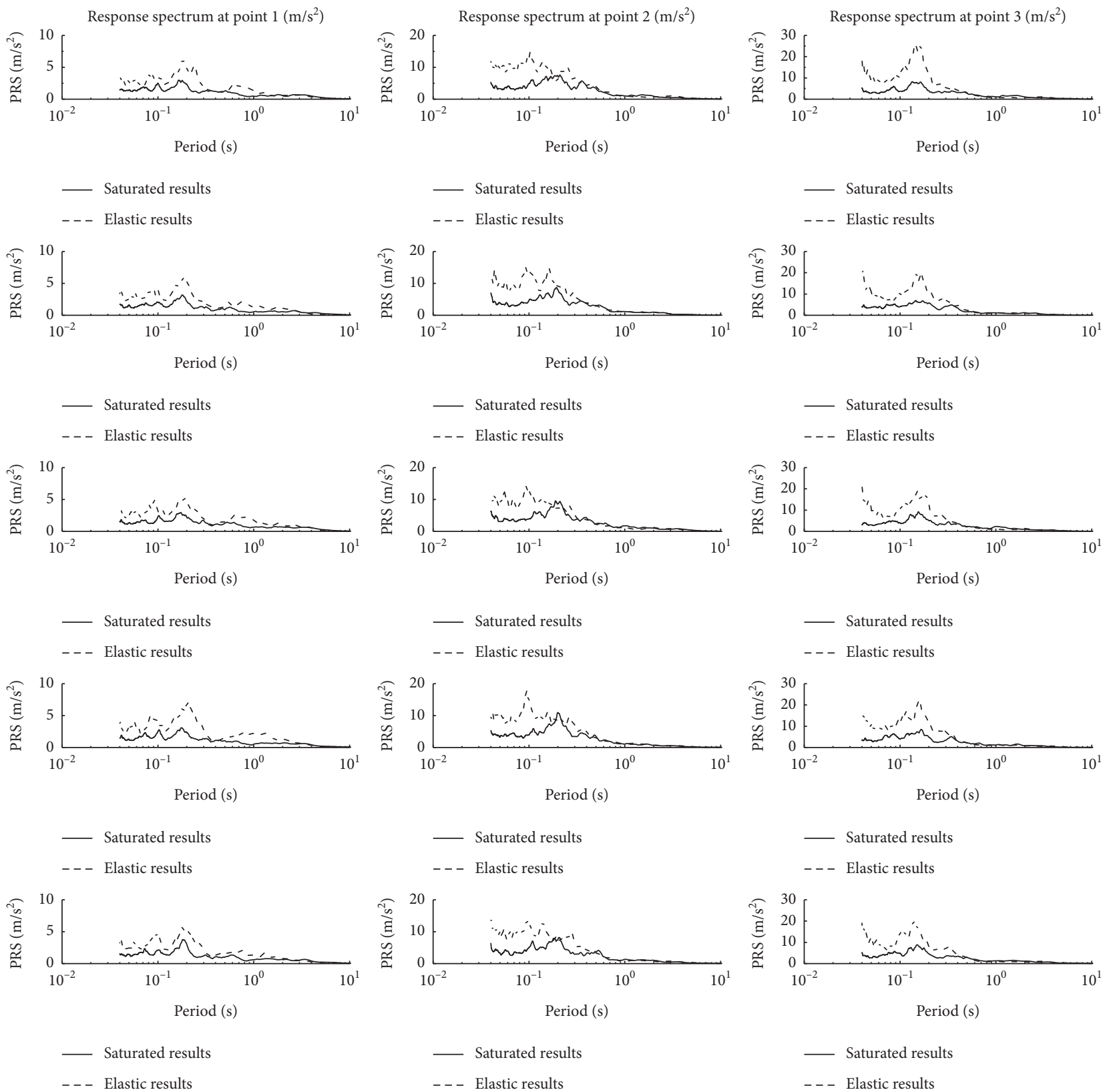

Figure 8: Comparison of ground motion response spectrum between two cases.

TABLE 2: Comparison of peak ground acceleration (PGA) and peak response spectrum (PRS) between the two cases.

\begin{tabular}{lccc}
\hline & Point 1 & Point 2 & Point 3 \\
\hline Maximum value of PGA1 $\left(\mathrm{m} / \mathrm{s}^{2}\right)$ & 0.97 & 2.75 & 2.78 \\
Maximum value of PGA2 $\left(\mathrm{m} / \mathrm{s}^{2}\right)$ & 1.77 & 4.43 & 5.64 \\
Mean value of PGA1 $\left(\mathrm{m} / \mathrm{s}^{2}\right)$ & 0.76 & 2.05 & 3.53 \\
Mean value of PGA2 $\left(\mathrm{m} / \mathrm{s}^{2}\right)$ & 1.37 & 11.48 & 4.31 \\
Maximum value of PRS1 $\left(\mathrm{m} / \mathrm{s}^{2}\right)$ & 4.21 & 18.81 & 14.63 \\
Maximum value of PRS2 $\left(\mathrm{m} / \mathrm{s}^{2}\right)$ & 7.05 & 4.14 & 28.42 \\
Mean value of PRS1 $\left(\mathrm{m} / \mathrm{s}^{2}\right)$ & 1.57 & 9.05 & 4.00 \\
Mean value of PRS2 $\left(\mathrm{m} / \mathrm{s}^{2}\right)$ & 2.96 & 0.18 & 10.36 \\
Saturated predominant period $(\mathrm{s})$ & 0.17 & 0.09 & 0.15 \\
Elastic predominant period $(\mathrm{s})$ & 0.18 & 0.10 \\
\hline
\end{tabular}



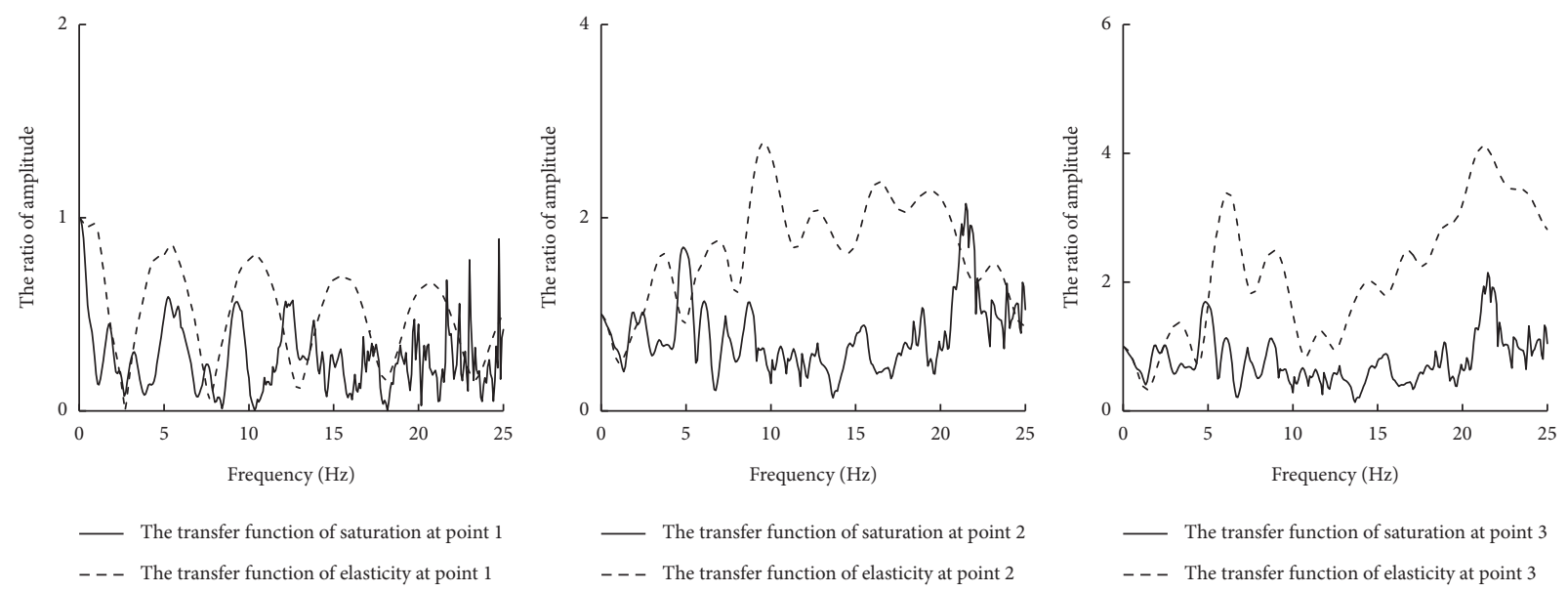

Figure 9: Comparison of the transfer function between two cases.

and from $0.10 \mathrm{~s}$ to $0.15 \mathrm{~s}$ at 3 point. The difference of periodic seismic response spectrum will affect the seismic response of aboveground structure to a great extent.

To further illustrate the difference between the saturated state and the elastic state, the transfer functions for both are shown in Figure 9. It can be seen that the high frequency response of the elastic model is more than that of the saturated model, and the high frequency response of the elastic model is overestimated. However, in the low-frequency region, especially, the seismic response of the long-period structures is underestimated, and the seismic disaster of the long-period structures in the alluvial valley is more serious. This is exactly what needs to be noted.

\section{Conclusions}

In this study, we proposed an efficient method for simulating spatially varying ground motions in a saturated alluvial valley based on Biot's theory, boundary element method, and spectral representation method. The feasibility of the simulation method was verified. A comparison among the results of the saturated model and elastic model revealed the following conclusions.

When the proposed multipoint ground motion simulation method is applied to the saturated deposition site, there is a good agreement between the target and theoretical values of the auto/cross-PSDs of the nonstationary random motion. The error is controlled within $5 \%$, which is less than the engineering standard error. It shows that the method has good applicability and strong feasibility in complex fields.

The comparison between the results of the saturation model and elastic model shows that the saturation property of saturated alluvial valley cannot be ignored. The characteristics of wave propagation in saturated two-phase media are essentially different from those in single-phase media, and there are complex fluid-solid coupling effects in the process of wave propagation. The fluid-saturated porous media model can more accurately reflect the actual formation information and can better meet the needs of the actual site response simulation in coastal and valley areas. For saturated soils with high porosity below the groundwater level or in coastal or valley areas, the single-phase model is difficult to describe the variation of pore pressure and flow rate, which will lead to large simulation errors. Especially, in the long-period range which is unfavorable to the long-span structure, the seismic response is underestimated.

For the simulation of spatially correlated multipoint ground motion over a saturated alluvial valley, it is necessary to consider the influence of saturated medium, local site scattering, and spatial coherence effect. The method presented in this paper can provide a new method for spatially related multipoint ground motion simulation of saturated complex site and provide a scientific basis for seismic fortification and seismic safety evaluation of actual complex valley site engineering, which has certain scientific significance and social benefits.

\section{Data Availability}

The basic data used to support the findings of this study are included within the article and available from the corresponding author upon request.

\section{Conflicts of Interest}

The authors declare that they have no conflicts of interest.

\section{Acknowledgments}

This research was supported by the National Natural Science Foundation of China (51908401), Tianjin Municipal Science and Technology Bureau (19PTZWHZ00080), and Tianjin "project + team" key training project (2020).

\section{References}

[1] M. D. Trifunac and D. E. Hudson, "Analysis of the pacoima dam accelerogram-san fernando, California, earthquake of 1971," Bulletin of the Seismological Society of America, vol. 61, no. 5, pp. 1393-1411, 1971.

[2] L. L. D. Lawrence and L. R. West, "Observed effects of topography on ground motion," Bulletin of the Seismological Society of America, vol. 63, no. 1, pp. 283-298, 1973. 
[3] J. G. Anderson, P. Bodin, J. N. Brune et al., "Strong ground motion from the michoacan, Mexico, earthquake," Science, vol. 233, no. 4768, pp. 1043-1049, 1986.

[4] S. Nolen-Hoeksema and J. Morrow, "A prospective study of depression and posttraumatic stress symptoms after a natural disaster: the 1989 loma prieta earthquake," Journal of Personality and Social Psychology, vol. 61, no. 1, pp. 115-121, 1991.

[5] S. E. Hough, J. R. Altidor, D. Anglade et al., "Localized damage caused by topographic amplification during the 2010 M 7.0 Haiti earthquake," Nature Geoscience, vol. 3, no. 11, pp. 778-782, 2010.

[6] J. Y. Han, M. Naggar, M. Zhao, Z. Zhong, B. Hou, and X. Du, "Longitudinal response of buried pipeline under non-uniform seismic excitation from multi-point shaking table tests," Soil Dynamics and Earthquake Engineering, vol. 140, no. 2, 2021.

[7] C. H. Loh, "Analysis of the spatial variation of seismic waves and ground movements from smart-1 array data," Earthquake Engineering \& Structural Dynamics, vol. 13, no. 5, pp. 561-581, 1985.

[8] Q. M. Feng and Y. X. Hu, "A mathematical model of spatially dependent ground motion," Journal of Earthquake Engineering and Engineering Vibration, vol. 1, no. 2, pp. 1-8, 1981, in Chinese.

[9] H. Hao, C. S. Oliveira, and J. Penzien, "Multiple-station ground motion processing and simulation based on SMART1 array data," Nuclear Engineering and Design, vol. 111, no. 3, pp. 293-310, 1989.

[10] R. Harichandran and E. H. Vanmarcke, "Stochastic variation of earthquake ground motion in space and time," Journal of Engineering Mechanics, vol. 112, no. 2, 1986.

[11] T. J. Qu, J. J. Wang, and Q. X. Wang, "A practical model of spatially varying ground motion power spectrum," Acta Seismologica Sinica (Chinese edition), vol. 18, no. 1, pp. 55-62, 1996, in Chinese.

[12] J. E. Luco and H. L. Wong, "Response of a rigid foundation to a spatially random ground motion," Earthquake Engineering \& Structural Dynamics, vol. 14, no. 6, pp. 891-908, 1986.

[13] P. G. Somerville, J. P. Mclaren, C. K. Saikia, and D. V. Helmberger, "Site-specific estimation of spatial incoherence of strong ground motion," in Earthquake Engineering and Structural Dynamics: II-Recent Advances in Ground Motion Evaluation, ASCE Geotechnical Special Publication, New York, NY, USA, no. 20, pp. 188-202, 1988.

[14] A. D. Kiureghian, "A coherency model for spatially varying ground motions," Earthquake Engineering and Structural Dynamics, vol. 25, no. 1, pp. 99-111, 1996.

[15] Z. X. Li, X. Q. Li, and N. Li, "Simulation of multi-point and multi-dimension spatially correlated earthquake ground motions," Earthquake Engineering and Engineering Dynamics, vol. 34, no. 4, pp. 64-72, 2014, in Chinese.

[16] L. J. Dou and B. P. Yang, "influence of site conditions on transfer function," Building Science, vol. 17, no. 3, pp. 40-46, 2001, in Chinese.

[17] J. W. Liang and Y. He, "A method of bedrock design spectrum inversion of engineering sites," Journal of Natural Disasters, vol. 28, no. 3, pp. 1-7, 2019, in Chinese.

[18] American Society of Civil Engineers (ASCE), Seismic Analysis of Safety-Related Nuclear Structures and Commentary, pp. 1118, American Society of Civil Engineers, Reston, VA, USA, 2003.

[19] M. D. Trifunac, "Scattering of plane SH waves by a semicylindrical canyon," Earthquake Engineering \& Structural Dynamics, vol. 1, no. 3, pp. 267-281, 1973.
[20] H. L. Wong, "Effect of surface topography on the diffraction of P, SV, and Rayleigh waves," Bulletin of the Seismological Society of America, vol. 72, no. 4, 1982.

[21] F. J. Sanchez-Sesma and M. Campillo, "Diffraction of P, SV, and Rayleigh waves by topographic features: a boundary integral formulation," Bulletin of the Seismological Society of America, vol. 81, no. 6, pp. 2234-2253, 1991.

[22] H. Cao and V. W. Lee, "Scattering and diffraction of plane P waves by circular cylindrical canyons with variable depth-towidth ratio," European Journal of Obstetrics \& Gynecology and Reproductive Biology, vol. 9, no. 3, pp. 141-150, 1979.

[23] Z. X. Liu, L. S. Zhu, and D. Wang, "Broad-band simulation of seismic response of alluvial valley using IBEM under incident plane P,SV, and Rayleigh waves," Chinese Quarterly of Mechanics, vol. 37, no. 3, pp. 58-71, 2017, in Chinese.

[24] Y. J. Chiou, D. C. Wong, J. C. Sung, and J. H. Jehng, “Application of the boundary element method to the scattering of $\mathrm{P}, \mathrm{SV}$, and Rayleigh waves by topographical irregularities," Journal of the Chinese Institute of Engineers, vol. 15, no. 2, pp. 167-178, 1992.

[25] M. I. Todorovska and V. W. Lee, "Surface motion of shallow circular alluvial valleys for incident plane waves-analytical solution," Soil Dynamics and Earthquake Engineering, vol. 10, no. 4, pp. 192-200, 1991.

[26] T. K. Mossessian and M. Dravinski, "Scattering of elastic waves by three-dimensional surface topographies," Wave Motion, vol. 11, no. 6, pp. 579-592, 1989.

[27] M. A. Biot, "Theory of propagation of elastic waves in a fluidsaturated porous solid. I. Low-Frequency range," Journal of the Acoustical Society of America, vol. 28, no. 2, pp. 168-178, 1956.

[28] C. H. Lin, Wave Propagation in a Poroelastic Half-Space Saturated with Inviscid Fluid, pp. 1-146, University of Southern California, Los Angeles, CA, USA, 2002.

[29] C. H. Lin, V. W. Lee, and M. D. Trifunac, "The reflection of plane waves in a poroelastic half-space saturated with inviscid fluid," Soil Dynamics and Earthquake Engineering, vol. 25, no. 3, pp. 205-223, 2005.

[30] W. H. Li and C. G. Zhao, "An analytical solution for the diffraction of plane $P$ waves by circular cylindrical canyons in a fluid-saturated porous media half space," Chinese Journal of Geophysics, vol. 46, no. 4, pp. 539-546, 2003, in Chinese.

[31] W. H. Li and C. G. Zhao, "Scattering of plane SV waves by cylindrical canyons in saturated porous medium," Soil Dynamics and Earthquake Engineering, vol. 25, no. 12, pp. 981-995, 2005.

[32] W. H. Li and C. G. Zhao, "Scattering of plane P waves in alluvial valleys with saturated soil deposits," Chinese Journal of Geotechnical Engineering, vol. 25, no. 3, pp. 346-351, 2003, in Chinese.

[33] W. H. Li and C. G. Zhao, "Scattering of plane SV waves by circular-arc alluvial valley s with saturated soil deposits," Chinese Journal of Geophysics, vol. 47, no. 5, pp. 911-919, 2004, in Chinese.

[34] Z. N. Ba, J. W. Liang, and V. W. Lee, "Diffraction of plane P waves by a shallow circular-arc canyon in a saturated poroelastic half-space," Journal of Tianjin University, vol. 43, no. 6, pp. 523-529, 2010, in Chinese.

[35] Z. X. Liu and J. W. Liang, "Diffraction of plane P waves around an alluvial valley in poroelastic half-space," Earthquake Science, vol. 23, no. 1, pp. 35-43, 2010.

[36] Y. He, Q. Yu, and Z. X. Liu, "Simulation of multi-point spatially correlated earthquake ground motions of sedimentary valleys considering scattering effect," Rock and Soil 
Mechanics, vol. 40, no. 7, pp. 2739-2747+2788, 2019, in Chinese.

[37] Y. He, Z. X. Liu, Q. Yu, and H. L. Wang, "Simulation of the spatially correlated multiple-station earthquake ground motions of the coupled alluvial valley-hill terrain," Engineering Analysis with Boundary Elements, vol. 118, pp. 41-53, 2020.

[38] Y. J. Chen, D. Y. Zhang, X. Li, H. Y. Jia, and W. M. Yan, "Stochastic seismic analysis of A cfst arch bridge under spatially varying ground motions," Engineering Mechanics, vol. 30 , no. 12 , pp. $99-106,2013$, in Chinese.

[39] R. B. Dong, "Nonlinear analysis of free spanning submarine pipelines subjected to spatially varying earthquake ground motions," China Civil Engineering Journal, vol. 41, no. 7, pp. 103-109, 2008, in Chinese.

[40] H. P. Jiang, Wang, and Q. S. Yang, "Behavior of long span rigid frame rc bridges subjected to spatial varying ground motion," Journal of Railway Engineering Society, vol. 18, no. 2, pp. 37-46, 2001, in Chinese.

[41] R. D. Xu and K. F. Zheng, "Analysis and improvement of simulation method for spatially correlative time histories of multi-point ground motions," Journal of Earthquake Engineering and Engineering Vibration, vol. 30, no. 2, pp. 10-15, 2010, in Chinese.

[42] M. Shrikhande and V. K. Gupta, "Synthesizing ensembles of spatially correlated accelerograms," Journal of Engineering Mechanics, vol. 124, no. 11, pp. 1185-1192, 1998.

[43] G. Deodatis, "Simulation of ergodic multivariate stochastic processes," Journal of Engineering Mechanics, vol. 122, no. 8, pp. 778-787, 1996.

[44] E. Heredia-Zavoni and S. Santa-Cruz, "Conditional simulation of a class of nonstationary space-time random fields," Journal of Engineering Mechanics, vol. 126, no. 4, pp. 398-404, 2000.

[45] R. Jankowski and K. Wilde, "A simple method of conditional random field simulation of ground motions for long structures," Engineering Structures, vol. 22, no. 5, pp. 552-561, 2000.

[46] E. H. Vanmarcke, E. Heredia-Zavoni, and G. A. Fenton, "Conditional simulation of spatially correlated earthquake ground motion," Journal of Engineering Mechanics, vol. 119, no. 11, pp. 2333-2352, 1993.

[47] R. W. Clough and J. Penzien, "Dynamics of structures," Journal of Applied Mechanics, vol. 44, no. 2, pp. 366, 1995.

[48] M. A. Biot, "Mechanics of deformation and acoustic propagation in porous media," Journal of Applied Physics, vol. 33, no. 4, pp. 1483-1498, 1962.

[49] Z. P. Liao, Introduction to Wave Motion Theories for Engineering, Science Press, Beijing, China, 2002, in Chinese.

[50] M. Dravinski and T. K. Mossessian, "Scattering of plane harmonic P, SV, and Rayleigh waves by dipping layers of arbitrary shape," Bulletin of the Seismological Society of America, vol. 77, no. 1, 1987. 